\title{
CONTROLE AUTONÔMICO DO CORAÇÃO E FRAÇÃO DE EJEÇÃO NA FASE CRÔNICA DO ACIDENTE VASCULAR ENCEFÁLICO
}

\author{
AUTONOMIC CONTROL OF THE HEART AND STROKE VOLUME IN THE CHRONIC PHASE OF \\ CEREBROVASCULAR ACCIDENT
}

\author{
CONTROL AUTONÓMICO DEL CORAZÓN Y FRACCIÓN DE EYECCIÓN EN LA FASE CRÓNICA \\ DEL ACCIDENTE VASCULAR ENCEFÁLICO
}

\begin{abstract}
Suenimeire Vieira' (Fisioterapeuta) Mônica Furquim de Campos ${ }^{1}$ (Fisioterapeuta)

Flávia Roberta Faganello Navega² (Fisioterapeuta)

Alexandre Ricardo Pepe Ambrozin² (Fisioterapeuta)

Aparecida Maria Catai ${ }^{2}$

(Fisioterapeuta)

Robison José Quitério ${ }^{3}$

(Fisioterapeuta)

1. Departamento de Educação Física, Instituto de Biociências, Universidade Estadual Paulista Júlio de Mesquita Filho, Campus de Rio Claro. SP, Brasil.

2-. Departamento de Fisioterapia e Terapia Ocupacional, Universidade Estadual Paulista Júlio de Mesquita Filho, Campus de Marília. SP, Brasil. 3. Departamento de Fisioterapia, Centro de Ciências Biológicas e da Saúde, Universidade Federal de São Carlos, São Carlos, SP, Brasil.
\end{abstract}

\section{Correspondência:}

Rua Nilton Silva, Jardim Santa Lúcia, 110, 14600-000. São Paulo, SP, Brasil. suenifv@hotmail.com

\section{RESUMO}

Introdução: Sujeitos acometidos por acidente vascular encefálico (AVE) podem apresentar alterações na estrutura e função do coração e na modulação autonômica. Objetivo: Verificar função autonômica e cardíaca sistólica em homens após AVE, e se estas variáveis estão associadas. Métodos: Foram avaliados oito homens, com idade entre 55 e 65 anos, acometidos por lesão cerebrovascular há pelo menos seis meses e todos com hemiparesia. Foram realizadas as seguintes avaliações: 1) Ecocardiografia, para avaliar a função cardíaca sistólica. 2) Registro da frequência cardíaca ( $F C$ ) e dos intervalos R-R (IRR), batimento a batimento, para avaliação do controle neural do coração. Esses dados foram analisados no domínio da frequência, por meio das análises dos seguintes componentes espectrais: alta frequência; baixa frequência e a razão BF/AF. Foi aplicado o teste de correlação de Pearson ( $p \leq 0,05)$. Resultados: Características demográficas, antropométricas e fisiológicas: Idade $=58,62 \pm 2,88$ anos; $I M C=27,41 \pm 5,33 \mathrm{~kg} / \mathrm{m}^{2} ; \mathrm{BF}=61,78 \pm 26,79$ (un); $\mathrm{AF}=38,23 \pm 26,79$ (un); $B F / A F=3,41 \pm 3,38 ; F E=0,65 \pm 0,04$. Não houve correlação estatisticamente significativa entre os índices espectrais BF e AF com fração de ejeção e a presença de fatores de risco para AVE. Conclusão: No presente estudo, a fração de ejeção do ventrículo esquerdo encontra-se normal e o controle neural do coração pode estar normal ou não, mesmo na presença de fatores de risco para o AVE.

Palavras-chave: controle da frequência cardíaca, ecocardiografia, hemiparesia.

\section{ABSTRACT}

Introduction: Subjects affected by cerebrovascular accident (CVA) may show changes in the structure and function of the heart and autonomic modulation. Objective: To assessautonomic and cardiac systolic function in men after stroke, and whether these variables are associated. Methods: Eight men aged between 55 and 65 years were evaluated, all suffering from cerebrovascular injury and with hemiparesis for at least six months. The following evaluations were performed: 1) echocardiography to evaluate systolic cardiac function; 2) Record the heart rate (HR) and RR intervals (RRI), beat by beat, to assess neural control of the heart. These data were analyzed in the frequency domain, by means of spectral analysis of the following components: high frequency, low frequency and LF/HF ratio. The Pearson correlation test was applied $(p \leq 0.05)$. Results: Demographic. anthropometric and physiological characteristics: Age $=58.62 \pm 2.88$ years; $B M I=27.41 \pm 5.33 \mathrm{~kg} / \mathrm{m}^{2} ; \mathrm{LF}=61.78 \pm 26.79$ (un); $\mathrm{HF}=38.23 \pm 26.79$ (un); $L F / H F=3.41 \pm 3.38 ; E F=0.65 \pm 0.04$. There was no statistically significant correlation between the spectral indices of low (LF) and high frequency (HF) with stroke volume and the presence of risk factors for CVA. Conclusion: In the present study, the stroke volume of the left ventricle is normal and the neural control of the heart may be normal or not, even in the presence of risk factors for CVA.

Keywords: heart rate control, echocardiography, paresis.

\section{RESUMEN}

Introducción: Los sujetos acometidos por accidente vascular encefálico (AVE) pueden presentar alteraciones en la estructura y función del corazón y en la modulación autonómica. Objetivo: Verificar función autonómica y cardíaca sistólica en hombres después de AVE, y si estas variables están asociadas. Métodos: Fueron evaluados ocho hombres, con edad entre 55 y 65 años, acometidos por lesión cerebrovascular desde hace por lo menos seis meses y todos con hemiparesia. Fueron realizadas las siguientes evaluaciones: 1) Ecocardiografía, para evaluar la función cardíaca sistólica. 2) Registro de la frecuencia cardíaca (FC) y de los intervalos R-R (IRR), latido a latido, para evaluación del control neural del corazón. Esos datos fueron analizados en el dominio de la frecuencia, a través del análisis de los siguientes componentes espectrales: alta frecuencia; baja frecuencia y la razón BF/AF. Fue aplicado el test de correlación de Pearson $(p \leq 0,05)$. Resultados: Características demográficas, antropométricas y fisiológicas: Edad $=58,62 \pm 2,88$ años; 
$I M C=27,41 \pm 5,33 \mathrm{~kg} / \mathrm{m}^{2} ; B F=61,78 \pm 26,79$ (un); $A F=38,23 \pm 26,79$ (un); $B F / A F=3,41 \pm 3,38 ; F E=0,65 \pm 0,04$. No hubo correlación estadísticamente significativa entre los índices espectrales BF y AF con fracción de eyección y la presencia de factores de riesgo para AVE. Conclusión: En el presente estudio, la fracción de eyección del ventrículo izquierdo se encuentra normal y el control neural del corazón puede estar normal o no, aún ante la presencia de factores de riesgo para el AVE.

Palabras clave: control de la frecuencia cardíaca, ecocardiografía, hemiparesia.

\section{INTRODUÇÃO}

O acidente vascular encefálico (AVE) representa a segunda maior causa de óbitos em todo o mundo (5,7 milhões de óbitos por ano)? No Brasil, está entre as principais causas, juntamente com as doenças crônicas não transmissíveis, ou seja, os transtornos neuropsiquiátricos, as doenças respiratórias crônicas, os cânceres e as doenças musculoesqueléticas ${ }^{2}$. Assim, o aumento da prevalência do AVE tem requerido maiores investigações sobre as suas repercussões orgânicas ${ }^{3,4}$.

A modulação do sistema nervoso autonômico (SNA) sobre o coração tem sido foco de pesquisas que têm revelado que após o AVE ocorre disfunção no sistema simpatovagal5,6 tanto na fase aguda quanto na fase crônica ${ }^{7}$, mesmo naqueles com boa recuperação física e cognitiva ${ }^{8}$. Porém, o nível de comprometimento dos eferentes simpático e parassimpático não está totalmente esclarecido. Enquanto alguns pesquisadores verificaram declínio na atividade parassimpática e aumento da atividade simpática ${ }^{6}$, outros observaram inibição parcial na atividade de ambos os eferentes autonômicos, na fase crônica do AVE⿳9 .

Essas divergências na literatura podem estar associadas à região lesada, ou seja, ao hemisfério cerebral e os níveis corticais e subcorticais acometidos $^{10,11}$. Lesões nos hemisférios cerebrais direito e esquerdo parecem prejudicar a atividade simpática e parassimpática, respectivamente ${ }^{10,12}$.

Para investigar a influência do SNA sobre o coração, a análise da variabilidade da frequência cardíaca (VFC) ${ }^{13,14}$ tem se mostrado um método reprodutível de grande relevância prognóstica que permite o diagnóstico precoce da disfunção autonômica e fornece informações adicionais para estratificação de risco, contribuindo, assim, para a tomada de medidas preventivas a fim de evitar complicações cardíacas ${ }^{5,6}$.

Está bem estabelecido que o sistema nervoso autônomo, por vezes prejudicado em indivíduos pós AVE, atua sobre a frequência cardíaca, volume sistólico, contratilidade, resistência vascular periférica e retorno venoso ${ }^{15}$. Desta forma, tem sido investigado se indivíduos pós-AVE podem apresentar anormalidades também na função cardíaca ${ }^{16}$, como lesão miocárdica mesmo na ausência de causas cardíacas primárias ${ }^{17}$ e alteração no enchimento do ventrículo esquerdo independente da presença de fatores de risco vasculares ${ }^{18}$.

Como as lesões nas estruturas corticais ou subcorticais verificadas após o AVE podem causar alterações no sistema nervoso autônomo ${ }^{19}$, mecanismo de controle extrínseco do coração, a hipótese é que indivíduos com lesão cerebrovascular apresentam menor VFC e diminuição na função cardíaca sistólica. Assim, o presente estudo tem como objetivo verificar função autonômica e cardíaca sistólica em indivíduos pós AVE em fase crônica, e ainda se essas variáveis estão associadas.

\section{MÉTODOS}

Foram triados setenta voluntários no serviço de neurologia da Clínica de Fisioterapia da Universidade e nas Unidades Básicas de Saúde do município de Marília, São Paulo, Brasil. porém apenas oito preencheram todos os critérios de inclusão e compuseram esse estudo: homens sedentários, não fumantes, com idade entre 55 e 65 anos, acometidos por lesão cerebrovascular há pelo menos seis meses e com hemiparesia. Foram excluídos aqueles que apresentaram: doenças respiratórias; disfunção neurológica e/ou cardiovascular prévia ao AVE; plegia em qualquer segmento corporal; distúrbios psiquiátricos e/ou cognitivos que impediriam a coleta dos dados; tabagistas.

O estudo foi transversal, aprovado pelo Comitê de Ética em Pesquisa com seres humanos (parecer 0093/2011). Depois de realizada a triagem, os voluntários foram informados sobre os objetivos do trabaIho e os procedimentos experimentais e assinaram o termo de consentimento livre e esclarecido conforme determina a resolução 196/96 e suas normas complementares do Conselho Nacional de Saúde.

Todas as avaliações foram realizadas no período da tarde em ambiente silencioso e climatizado $\left(23 \pm 2^{\circ} \mathrm{C}\right)$. Constaram de anamnese, coleta de dados sobre a história pregressa do voluntário, medidas da pressão arterial (PA) e frequência cardíaca ( $F C$ ), ausculta pulmonar e cardíaca, medidas da massa corporal e estatura, cálculo do índice de massa corpórea (IMC) ecocardiograma, registro da FC e dos intervalos RR.

A ecocardiografia transtorácica (Envisor $C^{\circledR}$, Philips, Estados Unidos) permitiu avaliar a fração de ejeção (FE), sendo considerados normais os valores iguais ou superiores a 0,5520.

A FC e os intervalos R-R (iR-R) instantâneos foram gravados por um sistema digital de telemetria validado previamente ${ }^{21}$ que consiste de um transmissor posicionado na altura do processo xifóide e um monitor / receptor (Polar RS800CX, Polar Electro Oy, Kempele, Finland). O sistema detecta a despolarização ventricular, que corresponde à onda R do eletrocardiograma, com frequência amostral de $500 \mathrm{~Hz}$ e resolução temporal de um milissegundo 22 .

Os voluntários não ingeriram café, chá, refrigerante e bebidas alcoólicas; mantiveram as atividades de vida diária nas 48 horas antes dos registros. O sujeito foi posicionado em decúbito dorsal e permaneceu em repouso até as variáveis fisiológicas estabilizarem (PA e FC). A partir desse momento e nessa postura foi realizado o registro da FC e dos intervalos RR durante 10 minutos.

Os dados foram transmitidos para um computador utilizando-se o software Polar Precision Performance (versão 3.02.007) e convertidos em arquivos de texto que foram analisados no Software Kubios HRV (versão 2.0, University of Kuopio, Finland). Foi aplicado filtro digital para eliminar os intervalos RR anormais e selecionado o trecho mais estável para análise, sempre considerando 256 pontos. Para o cálculo do espectro foi aplicado o método de interpolação splines cúbicas com frequência de $4 \mathrm{~Hz}$ e os dados foram processados aplicando-se a Transformada Rápida de Fourier (FFT), que decompôs o sinal nas seguintes bandas: alta frequência ( $\mathrm{AF}-0,15 \mathrm{a} 0,4 \mathrm{~Hz}$ ) que se associa a atividade parassimpática; baixa frequência (BF - 0,04 a 0,15Hz) que representa atividade simpática e parassimpática, porém com o predomínio da simpática; e a razão BF/AF que representa o balanço simpatovagal13,14. Os índices de VFC foram apresentados em unidades normalizadas (un) calculados da seguinte maneira: divisão da densidade espectral de potência de uma dada banda (i.e. BF ou AF) pela potência total, subtraindo a banda de muito baixa frequência e multiplicando por $100^{23}$.

\section{Análise Estatística}

Os dados demográficos, antropométricos e fisiológicos são apresentados de forma descritiva em média e desvio padrão. Foi aplicado 
o teste de normalidade Kolmogorov-Smirnov, e verificado distribuição normal dos dados. Para testar a hipótese de existência de associação entre os índices de VFC com a fração de ejeção, foi aplicado o teste de correlação de Pearson. O nível de significância adotado foi de 5\% $(p \leq 0,05)$. Foi utilizado o software de análise GraphPad InStat ${ }^{\circledR}$.

Para testar a hipótese de existência de associação entre os índices de variabilidade da frequência cardíaca e a presença de fatores de risco e uso de medicamentos foi aplicado o Teste exato de Fisher. Foram considerados como valores prejudicados (de corte) BF $\geq 58$ e AF $\leq 26(14,25)$.

O cálculo amostral foi realizado por meio do software Ene ${ }^{\circledR}$, versão 3.0 (Barcelona, Espanha). Considerou-se a correlação entre as variáveis AF e fração de ejeção realizada em estudo piloto. Assim, utilizou-se $r=0,62$, poder estatístico de $80 \%$ e alfa de 0,05. Com estes parâmetros, determinou-se um tamanho de amostra de 15 voluntários.

\section{RESULTADOS}

A amostra consistiu de oito homens, sendo sete com hemiparesia direita e um com hemiparesia esquerda. As características demográficas, antropométricas e fisiológicas são apresentadas na tabela 1 e os fatores de risco e medicamentos vigentes, na tabela 2.

Não houve correlação estatística significativa entre os índices espectrais de baixa (BF) e alta frequência (AF) de VFC com fração de ejeção (figuras 1,2 e 3) e com a presença de fatores de risco para AVE.

Tabela 1. Características demográficas, antropométricas e fisiológicas de homens hemiparéticos $(\mathrm{N}=8)$.

\begin{tabular}{|c|c|c|}
\hline & Média \pm Desvio Padrão & Valores de referência \\
\hline Idade (anos) & $58,62 \pm 2,88$ & \\
\hline Estatura $(\mathrm{m})$ & $1,68 \pm 0,07$ & \\
\hline Massa $(\mathrm{kg})$ & $77,36 \pm 14,92$ & \\
\hline IMC $\left(\mathrm{kg} / \mathrm{m}^{2}\right)$ & $27,41 \pm 5,33$ & 18,5 a 25 \\
\hline PAS $(\mathrm{mmHg})$ & $123,75 \pm 14,08$ & 100 a 140 \\
\hline PAD $(\mathrm{mmHg})$ & $76,25 \pm 7,44$ & 60 a 90 \\
\hline $\mathrm{BF}$ (un) & $61,78 \pm 26,79$ & 50 a 58 \\
\hline $\mathrm{AF}$ (un) & $38,23 \pm 26,79$ & 26 a 39 \\
\hline $\mathrm{BF} / \mathrm{AF}$ & $3,41 \pm 3,38$ & \\
\hline $\mathrm{FC}(1 / \mathrm{min})$ & $71,86 \pm 14,57$ & \\
\hline IRR (ms) & $865,43 \pm 155,88$ & \\
\hline Fração de Ejeção & $0,65 \pm 0,04$ & $\geq 0,55$ \\
\hline Tempo após AVE (anos) & $4,5 \pm 3,3$ & \\
\hline Colesterol Total (mg/dl) & $209,75 \pm 47,50$ & $<200$ \\
\hline Colesterol HDL (mg/dl) & $43,13 \pm 8,97$ & 19 anos: $\geq 50$ \\
\hline Triglicerideos (mg/dl) & $273,75 \pm 249,73$ & Normal: $₫ 150$ \\
\hline Glicemia (mg/dl) & $113,63 \pm 63,20$ & Entre 75-99 \\
\hline
\end{tabular}

m: metros; Kg: Quilogramas; kg/m²: Quilogramas por metros ao quadrado; IMC: índice de massa corporal; PAS: Pressão Arterial Sistólica: PAD: Pressão Arterial Diastólica: $\mathrm{mmHg}$ : milímetros de mercúrio; BF: índice de baixa frequência; AF: índice de alta frequência; um: unidades normalizadas; FC: Frequencia cardíaca; IRR: Intervalos RR; ms: milissegundos; mg/dl: miligrama por decilitro; AVE: acidente vascular encefálico.

Tabela 2. Fatores de risco para doença cardiovascular e medicamentos em uso no momento do estudo.

\begin{tabular}{c|c|c}
\hline & Voluntários $(\mathbf{n})$ & Voluntários (\%) \\
\hline Fatores de risco & & \\
\hline Dislipidemia & 5 & 62,5 \\
\hline Hiperglicemia & 3 & 37,5 \\
\hline Hipertensão & 6 & 75 \\
\hline Medicamentos & & \\
\hline ácido acetilsalićlico & 5 & 62,5 \\
\hline sinvastatina & 5 & 62,5 \\
\hline maleato de enalapril & 2 & 25 \\
\hline losartana potássica & 2 & 25 \\
\hline captopril & 2 & 25 \\
\hline n: número de voluntários & &
\end{tabular}

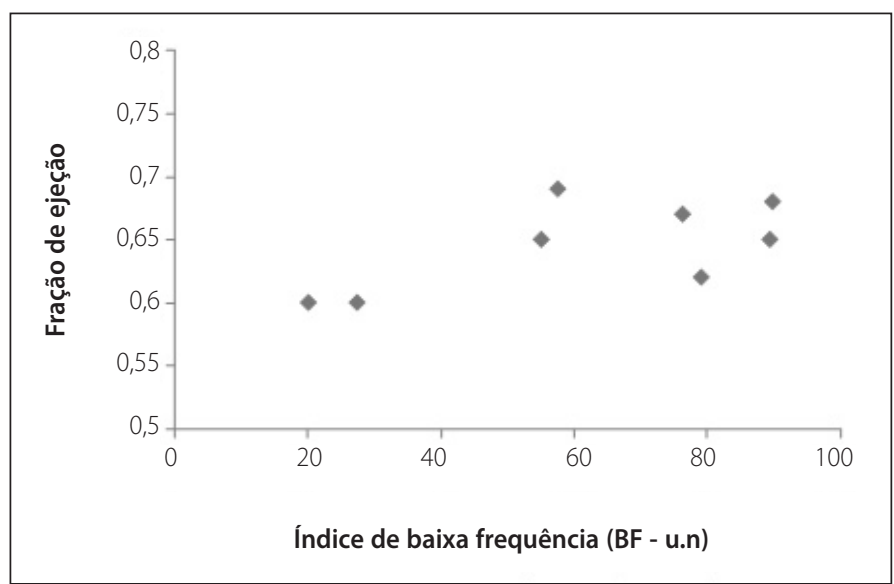

Figura 1. Dispersão dos dados de índice espectral de baixa frequência (BF - 0,04 a $0,15 \mathrm{~Hz})$ e fração de ejeção em homens hemiparéticos ( $N=8) r=0,65 ; p=0,08$.

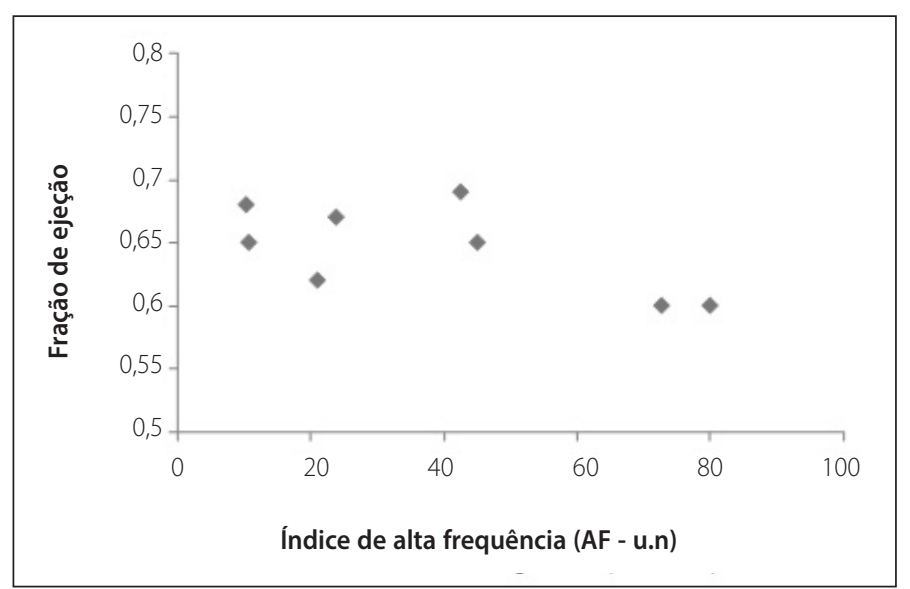

Figura 2. Dispersão dos dados de índice espectral de alta frequência (AF - 0,15 a $0,4 \mathrm{~Hz})$ e fração de ejeção em homens hemiparéticos $(N=8) r=-0,65 ; p=0,08$.

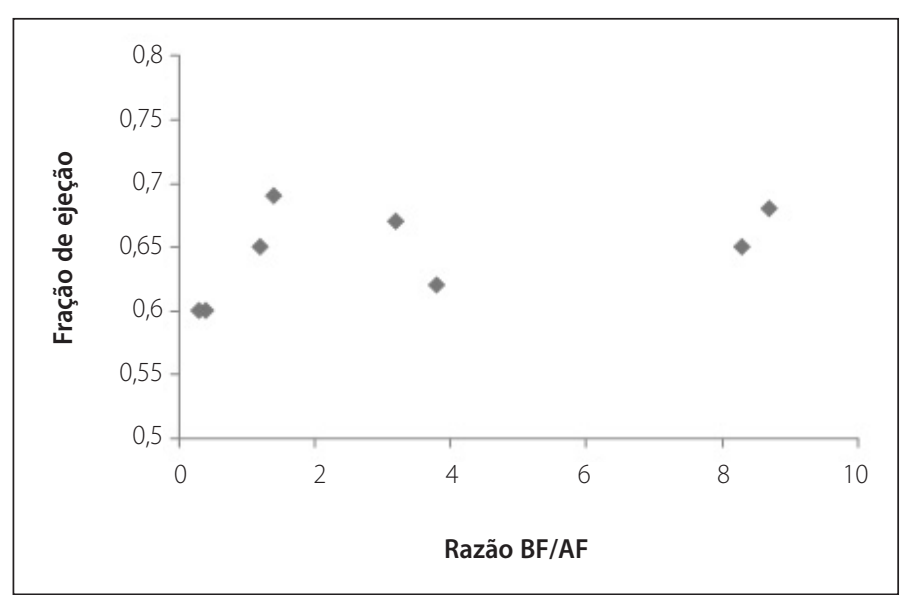

Figura 3. Dispersão dos dados de Razão BF/AF e fração de ejeção em homens hemiparéticos $(N=8) r=0,45 ; p=0,27$.

\section{DISCUSSÃO}

A literatura refere como normais os valores dos índices espectrais de variabilidade da frequência cardíaca, em unidades normalizadas, entre 50 a 58 para o índice de baixa frequência (BF), que representa a atividade de ambos os eferentes autonômicos, com predomínio simpático, e entre 26 a 39 para alta frequência, que representa a atividade parassimpática ${ }^{14,24}$.

No presente estudo, metade da amostra apresentou valores normais relacionados à modulação parassimpática e simpática, enquanto os demais tiveram os índices dos aferentes prejudicados. 
Estudos a esse respeito têm observado declínio na atividade parassimpática e aumento da atividade simpática ${ }^{6}$ ou inibição parcial na atividade de ambos os eferentes autonômicos após o AVE em fase crônica ${ }^{9}$. Estas adaptações são atribuídas às alterações vasculares das grandes e pequenas artérias cerebrais ${ }^{25}$ e consequentemente, à área e hemisférios cerebrais acometidos, responsáveis ou não pelo controle autonômico do coração ${ }^{11}$ e considera-se o córtex insular a mais importante área cortical no controle simpatovagal, devido às extensas conexões com outras áreas reguladoras do sistema nervoso autonômico ${ }^{10,19}$. Na presente pesquisa sete voluntários apresentaram lesão no hemisfério esquerdo e um no hemisfério direito. Portanto, esses resultados obtidos discordam daqueles que afirmam que as lesões nos hemisférios cerebrais direito e esquerdo prejudicam a atividade simpática e parassimpática ${ }^{12,10}$, respectivamente, já que metade dos voluntários estudados apresentou valores referidos como normais ${ }^{14,24}$ e os demais tiveram alteração tanto no simpático, quanto no parassimpático.

Por outro lado, nossos resultados divergem, em parte, daqueles que referem que na fase crônica do AVE não há alterações na modulação autonômica ${ }^{7}$. Tais divergências podem estar relacionadas ao fato dessas adaptações caracterizarem uma situação transitória ${ }^{27}$ e/ou estarem relacionadas à independência funcional, ou seja, é possível que indivíduos mais independentes recuperam a VFC mais rapidamente ${ }^{28}$. Além disso, tem sido verificado que a presença de fatores de risco tradicionais para o AVE pode prejudicar a modulação autonômica cardíaca ${ }^{26}$, entretanto, não foi encontrada associação entre os índices de baixa (BF) e alta frequência (AF) com dislipidemia, hiperglicemia, obesidade e hipertensão presentes nos sujeitos estudados.

No que se refere à hipótese de que a lesão cerebrovascular está associada às adaptações na função cardíaca ${ }^{6,16}$ não foi confirmada no presente estudo, onde se verificou que a fração de ejeção, medida para expressar a integralidade da contração cardíaca e de extrema valia na apreciação objetiva da função ventricular sistólica, apresentou valores normais ${ }^{20}$, assim como constataram Masugata et al..$^{30}$. Porém, difere de McAreavey et al. ${ }^{18}$ que constataram que a alteração no enchimento do ventrículo esquerdo

\section{REFERÊNCIAS}

1. Soler EP, Ruiz VC. Epidemiology and Risk Factors of Cerebral Ischemia and Ischemic Heart Diseases: Similarities and Differences. Curr Cardiol Rev. 2010;6(3):138-49.

2. Schmidt MI, Duncan BB, Azevedo e Silva G, et al. Chronic non-communicable diseases in Brazil: burden and current challenges. Lancet. 2011;4:377(9781):1949-61.

3. Zorowitz RD, Gross E, Polinski DM. The stroke survivor. Disabil Rehabil. 2002;24(13):666-79.

4. Elkind MS. Stroke in the elderly. Mt Sinai J Med. 2003;70(1):27-37.

5. Günther A, Witte OW, Hoyer D. Autonomic Dysfunction and Risk Stratification Assessed from Heart Rate Pattern. Open Neurol J. 2010;4:39-49.

6. Hilz MJ, Moeller S, Akhundova A, Marthol H, Pauli E, De Fina P, et al. High NIHSS values predict impairment of cardiovascular autonomic control. Stroke. 2011;42(6):1528-33.

7. Lakusic N, Mahovic D, Babic T. Gradual recovery of impaired cardiac autonomic balance within first six months after ischemic cerebral stroke. Acta Neurol Belg. 2005;105(1):39-42.

8. Mclaren A, Kerr S, Allan L, Steen IN, Ballard C, Allen J, et al. Autonomic Function Is Impaired in Elderly Stroke Survivors. Stroke. 2005;36(5):1026-30.

9. Muslumanoglu L, Akyuz G, Aki S, Karsidag S, Us O. Evaluation of autonomic nervous system functions in post-stroke patients. Am J Phys Med Rehabil. 2002;81(10):721-5.

10. Oppenheimer SM, Gelb A, Girvin JP, Hachinski VC. Neurology. Cardiovascular effects of human insular cortex stimulation. 1992;42(9):1727-32.

11. He L, Li C, Luo Y, Dong W, Yang H. Clinical prognostic significance of heart abnormality and heart rate variability in patients with stroke. Neurol Res. 2010;32(5):530-4.

12. Lane RD, Wallace JD, Petrosky PP. Supraventricular tachycardia in patients with right hemisphere strokes. Stroke.1992;23:362-6.

13. Longo A, Daniel F, Correia MJ. Variabilidade da frequência cardíaca. Revista Portuguesa de Cardiologia. 1995; 14(3):241-62.

14. Task force of the European Society of Cardiology of the North American Society of Pacing Electrophysiology. Heart Rate Variability Standards of Measurement, Physiological Interpretation, and Clinical Use. Circulation. 1996;93(5):1043-65

15. Irigoyen MC, Consolim-Colombo FM, Krieger EM. Controle cardiovascular: regulação reflexa e papel do sistema nervoso simpático. Rev Bras Hipertens. 2011;8(1):55-62.

16. Min J, Farooq MU, Greenberg E, Aloka F, Bhatt A, Kassab M, et al. Cardiac Dysfunction After Left Perma- apresentou associação forte com o infarto cerebral em idosos após o AVE, e esta relação foi independente dos fatores de risco vasculares.

As limitações do estudo referem-se ao número de sujeitos da amostra que ficou abaixo do indicado no cálculo amostral em virtude das dificuldades em encontrar pessoas que preenchessem os critérios de seleção; a impossibilidade de acesso ao exame diagnóstico do AVE para melhor determinar a área de lesão; a falta de um grupo controle com o perfil similar ao estudado, porém sem terem sofrido AVE; e a não inclusão de uma metodologia de análise da independência funcional para investigar se as adaptações autonômicas cardíacas estão relacionadas a mesma.

\section{CONCLUSÃO}

Concluímos que na fase crônica do AVE a fração de ejeção do ventrículo esquerdo encontra-se normal e o controle neural do coração pode estar normal ou não, mesmo na presença de fatores de risco para o AVE. E ainda, que não existe associação entre essas duas últimas variáveis e entre função sistólica cardíaca e os índices de VFC.

Clinicamente, é importante conhecer que a VFC é um método confiável, de baixo custo e de grande relevância prognóstica que permite o diagnóstico precoce da disfunção autonômica cardíaca, associada a fatores de risco cardiovasculares, dentre os quais, o acidente vascular encefálico.

\section{AGRADECIMENTOS}

Ao Instituto do Coração (ICM); Ao Laboratório de Análise Clínicas São Francisco; Aos órgãos de fomento Conselho Nacional de Desenvolvimento Científico e Tecnológico (CNPq) e Fundação para o Desenvolvimento da UNESP (Fundunesp); Ao Centro de Educação e Estudos em Saúde (CEES); Secretaria de Saúde Pública do Município de São Paulo.

Todos os autores declararam não haver qualquer potencial conflito de interesses referente a este artigo. nent Cerebral Focal Ischemia: The Brain and Heart Connection. Stroke. 2009;40(7):2560-3.

17. Ay H, Koroshetz WJ, Benner T, Vangel MG, Melinosky C, Arsava EM, et al. Neuroanatomic correlates of stroke-related myocardial injury. Neurology. 2006;66(9):1325-9.

18. McAreavey D, Vidal JS, Aspelund T, Owens DS, Hughes T, Garcia M, et al. Correlation of Echocardiographic Findings with Cerebral Infarction in Elderly Adults: The AGES-Reykjavik Study. Stroke. 2010;41(10):2223-8.

19. Oppenheimer SM, Cechetto DF. Cardiac chronotropic organization of the rat insular córtex. Brain Research. 1990;533(1):66-72.

20. European Association of Echocardiography, a branch of the European Society of Cardiology. J Am Soc Echocardiogr. 2005;18(12):1440-63

21. Gamelin FX, Berthoin S, Bosquet L. Validity of Polar $\mathbf{S} 810$ Heart Rate Monitor to Measure R-R Intervlas at Rest. Med Sci Sports Exerc. 2006;38(5):887-93.

22. Ruha A, Sallinen S, Nissila S. A real-time microprocessor QRS detector system with a 1 ms timing accuracy for the measurement of ambulatory HRV. IEEE Trans Biomed Eng. 1997;44(3):159-67.

23. Malliani A. The Pattern of Sympathovagal Balance Explored in the Frequency Domain. Physiology. 1999;14(3):111-7.

24. Nunan D, Sandercock GRH, Brodie DA. A Quantitative Systematic Review of Normal Values for ShortTerm Heart Rate Variability in Healthy Adults. Pacing Clin Electrophysiol.; 2010;33(11):1407-17.

25. Chen CF, Lai CL, Lin HF. Reappraisal of heart rate variability in acute ischemic Stroke. Kaohsiung Journal of Medical Sciences. 2011;27(6):215-21.

26. Shehab A, Elnour AA, Struthers AD. Heart rate variability as an indicator of left ventricular systolic dysfunction. Cardiovasc J Afr. 2009;20(5):278-83.

27. Meglic B, Kobal J, Osredkar J, PogacnikT. Autonomic nervous system function in patients with acute brainstem stroke. Cerebrovasc Dis. 2001;11(1):2-8.

28. Bassi A, Colivicchi F, Santini M, Caltagirone C. Cardiac autonomic dysfunction and functional outcome after ischaemic stroke. European Journal of Neurology. 2007;14(8):917-22.

29. Korpelainen JT, Sotaniemi KA, Huikuri HV, Myllyä VV. Abnormal Heart Rate Variability as a Manifestation of Autonomic Dysfunction in Hemispheric Brain Infarction. Stroke. 1996;27(11):2059-63.

30. Masugata H, Senda S, Goda F. Cardiac diastolic dysfunction is associated with cerebral white matter lesions in elderly patients with risk factors for atherosclerosis. Tohoku J. Exp. Med. 2008;216(2):99-108 